\title{
Salvage strategy for long-term central venous catheter- related Staphylococcus aureus infections in children
}

Fanny Alby-Laurent ${ }^{1}$, Cécile Lambe ${ }^{2}$, Agnès Ferroni ${ }^{3}$, Nadège Salvi ${ }^{4}$, David Lebeaux ${ }^{5}$, Martin Castelle ${ }^{6}$, Florence Moulin ${ }^{7}$, Xavier Nassif ${ }^{3}$, Olivier Lortholary ${ }^{5}$, Martin Chalumeau ${ }^{1}$ and Julie Toubiana ${ }^{1}$

${ }^{1}$ Dpt. of Pediatrics and Infectious Diseases; ${ }^{2}$ Dpt. of Pediatric Gastroenterology, ${ }^{3}$ Dpt. of Clinical Microbiology; ${ }^{4}$ Dpt. of Pediatric Critical Care and Anesthesia; ${ }^{5}$ Necker-Pasteur Center for Infectious Diseases \& Tropical Medicine; ${ }^{6}$ Pediatric Hematology-Immunology Unit; ${ }^{7}$ Dpt. of Pediatric Intensive Care Unit. AP-HP, Necker-Enfants malades University Hospital, Paris Descartes University, Paris, France

\section{Background}

- The use of long-term central venous catheter (CVC) in children is associated with serious complications such as central-line associated bloodstream infection (CLABSI) (1)

- Current international guidelines strongly recommend catheter removal in case of $S$. aureus CLABSI, as it is associated with high risk of failure or complications (2)

- Pediatricians are however often tempted to have a catheter salvage strategy (CSS) because of the inconvenience of catheter replacement in children

- No data is available regarding the outcome of CSS in children

\section{Methods}

- Retrospective observational study from 2010 to 2014 in a tertiary care teaching hospital

- Inclusion of all children $<18$ years having a probable or proven S. aureus CLABSI on a long-term CVC

- CSS was defined as CVC left in place $\geq 3$ days after initiation of empiric treatment for suspected bacteremia

- Failure was defined as persistence or relapse of bacteremia with the same microbial species, or the occurrence or the worsening of local or systemic infectious complication between 72 hours and 28 days after the first positive blood culture

\section{Results}

- 49 S. aureus CLABSI were observed in 41 children (Figure), sex ratio (M/F) was 0.70 , and median age at the time of infection 1.4 years [0.1-16.9]

- 44 patients (59\%) had a digestive pathology requiring parenteral nutrition, 14 (34\%) had an immune deficiency

- CSS was chosen in $37(76 \%)$ cases and failed in $12(32 \%)$ due to persistence/relapse of bacteremia, thrombophlebitis, endocarditis or tunnel infection

- Initial presence of bloodstream co-infection, serum concentration of vancomycin under the targeted value, and inadequate empiric treatment were statistically significantly associated with CSS failure $(p<0.05)($ Table)

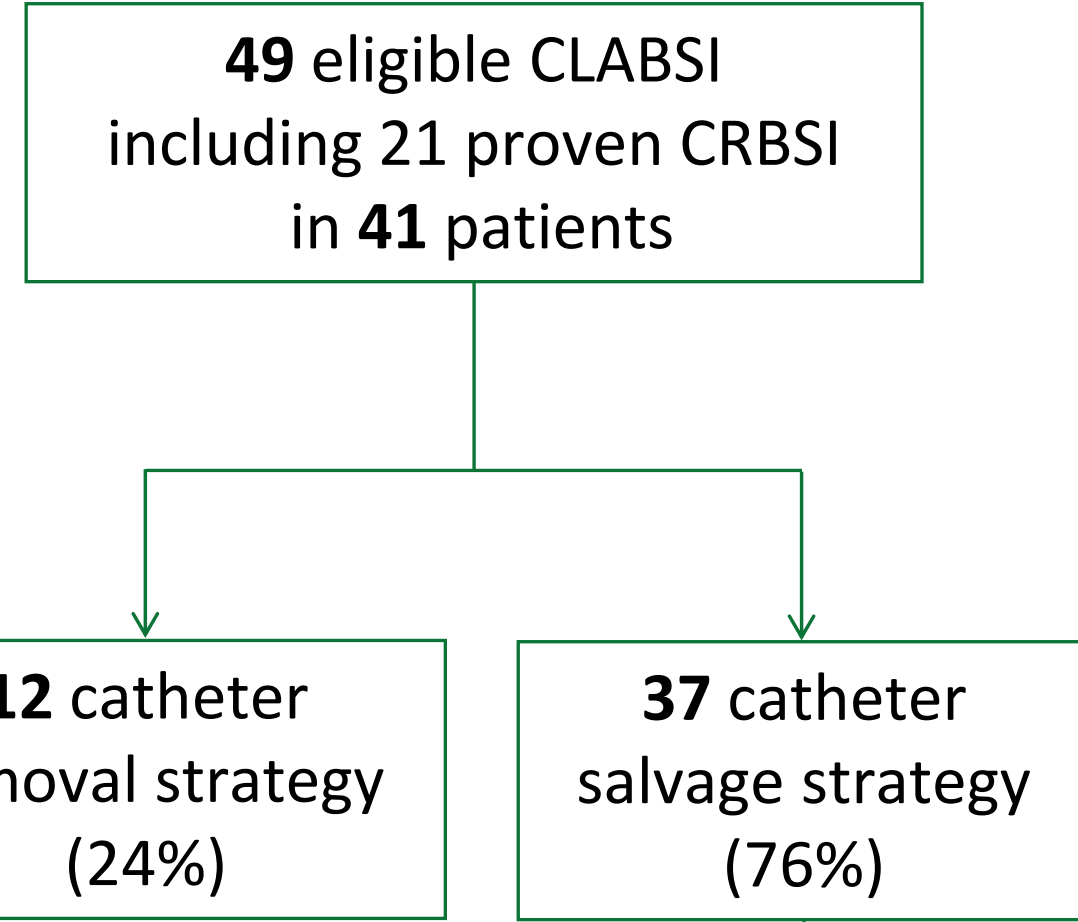

removal strategy

$(24 \%)$

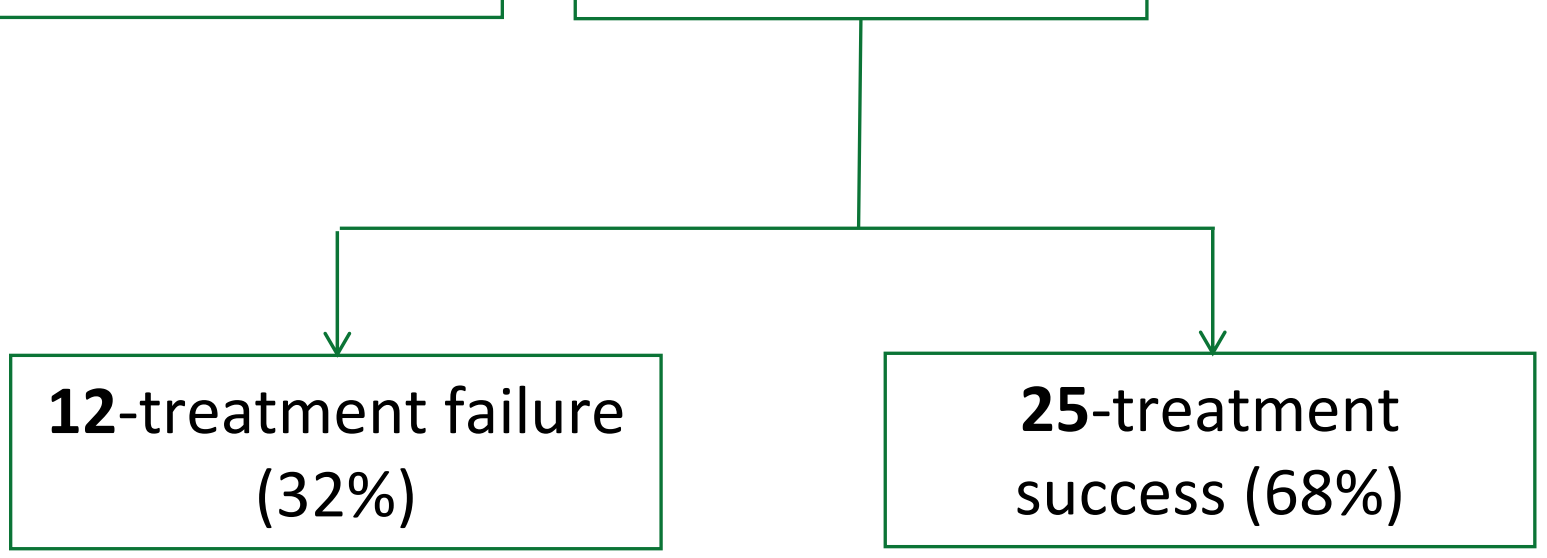

Figure. Flow chart of $S$. aureus CLABSI

\begin{tabular}{|c|c|c|c|}
\hline & $\begin{array}{c}\text { Failure (\%) } \\
n=12\end{array}$ & $\begin{array}{l}\text { Success (\%) } \\
\qquad n=25\end{array}$ & P value \\
\hline \multicolumn{4}{|l|}{ General characteristics } \\
\hline Age, year; median [range] & $1.5[0.3-6.9]$ & $1.6[0.2-16.9]$ & 0.81 \\
\hline Male & $5(42)$ & $9(36)$ & 0.74 \\
\hline Immunosuppression & $4(33)$ & $7(28)$ & 0.74 \\
\hline Tunneled \pm sleeved catheter & $10(83)$ & $22(88)$ & 0.70 \\
\hline Days of catheter use; median [range] & $28[2-548]$ & $15[0-468]$ & 0.25 \\
\hline \multicolumn{4}{|l|}{ Infectious episode } \\
\hline Local symptoms at onset & $4(33)$ & $7(28)$ & 0.74 \\
\hline Severe sepsis at onset & $4(33)$ & $2(8)$ & 0.05 \\
\hline CRP at onset (mg/L) median; [range] & $57[7-165]$ & $73[6-245]$ & 0.76 \\
\hline Polymicrobial infection & $5(42)$ & $3(12)$ & 0.04 \\
\hline S. aureus methicillin resistant strain* & $2(17)$ & $4(16)$ & 0.96 \\
\hline \multicolumn{4}{|l|}{ Empiric treatment } \\
\hline Cloxacillin & $0(0)$ & $1(4)$ & 0.51 \\
\hline $\begin{array}{l}\text { Adapted vancomycin serum } \\
\text { concentration }\end{array}$ & $2(29)$ & $13(72)$ & 0.04 \\
\hline Combined therapy & $4(33)$ & $10(40)$ & 0.69 \\
\hline Antibiotic locks & $2(17)$ & $2(8)$ & 0.43 \\
\hline Repair kit & $3(25)$ & $2(8)$ & 0.16 \\
\hline Inadequate empiric treatment & $2(17)$ & $0(0)$ & 0.04 \\
\hline
\end{tabular}

Table. Factors associated with the failure of a conservative therapy

\section{Conclusion}

- This is the first observational study focusing on long-term S. aureus CLABSI management in children.

- We reported that CSS for long-term CVC-related S.aureus infections was frequent (76\%) in a French university hospital and frequently successful 